Voix et Images

volxetimages

\title{
Les romanciers du XIXe siècle face à leurs romans : Notes pour la reconstitution d'une argumentation
}

\section{Javier Garcia-Mendez}

Volume 8, numéro 2, hiver 1983

Marie-Claire Blais

URI : https://id.erudit.org/iderudit/200386ar

DOI : https://doi.org/10.7202/200386ar

Aller au sommaire du numéro

Éditeur(s)

Les Presses de l’Université du Québec

ISSN

0318-9201 (imprimé)

1705-933X (numérique)

Découvrir la revue

Citer cet article

Garcia-Mendez, J. (1983). Les romanciers du XIXe siècle face à leurs romans :

Notes pour la reconstitution d'une argumentation. Voix et Images, 8(2),

331-343. https://doi.org/10.7202/200386ar d'utilisation que vous pouvez consulter en ligne.

https://apropos.erudit.org/fr/usagers/politique-dutilisation/ 


\title{
Les romanciers du XIXe siècle face à leurs romans: Notes pour la reconstitution d'une argumentation'
}

\author{
par Javier Garcia-Mendez. Université du Québec à Montréal
}

Dans les années soixante du siècle dernier. Casgrain prépare la réédition du roman que Philippe Aubert de Gaspé fils avait publié en 1837. Ce partisan d'une littérature nationalé agrave, méditative, spiritualiste, religieuse, évangélisatrice comme nos missionnaires $n^{2}$ s'adonne alors à une opération de remaniement qui ne dédaigne pas la suppression de passages complets du texte original. II s'agissait de donner à l'imprimerie un roman ad usum hominis canadiensis. un roman adéquat aux Canadiens français de l'époque tels que le groupe social dont l'abbé était l'un des idéologues se les représentait. On sait que la sévère et laborieuse main du religieux s'attarda même au titre original de ce roman, lui préférant celui de le Chercheur de trésors. Fort heureusement, le geste purificateur ne nous a pas privés de connaitre le véritable titre; un titre qui revêt une importance toute singulière car, dans la mesure où le livre du jeune de Gaspé passe pour être le premier roman écrit au Québec, les mots qu'il énonce à son début peuvent être tenus pour le premier vagissement du genre romanesque dans ce pays.

Placé dans un lieu mitoyen entre la réalité et l'imaginaire, le titre de tout roman participe à la fois de l'événementiel quotidien et de l'événementiel fictionnel. Sa situation n'implique pas encore cette mise entre parenthèses transitoire de la réalité immédiate qui est le propre de la lecture fictionnelle: cependant, c'est à partir de lui que le déploiement de la fiction s'opère. Frontière entre la réalité et l'écriture romanesque, le titre du premier roman québécois, I'Influence d'un livre. commence par nommer et l'écriture et la réalité. La première est présente dans le mot livre; peu importe que le texte dont il est question ici, Je Petit Albert, ne soit pas un roman; ce qui est à retenir c'est la référence, aux portes d'entrée d'une écriture. a l'écriture, le renvoi du livre a lui-même par cette présence du mot livre dans le titre. Mais celui-ci ne s'occupe du livre que de manière secondaire; son thème n'est pas tant l'ouvrage d'alchimie auquel il réfère mais son influence sur quelqu'un, l'effet concret. sensible de l'écrit sur la réalité. Voilà la façon dont le titre, élément situé aux frontières du réel et du littéraire, nomme, dans le roman de Gaspé fils, les deux versants dont il occupe la zone de confluence. Mais de ces deux versants. 
c'est celui de la réalité qui est privilégié. En retenant du livre non quelque chose se trouvant en son ıntérıeur mais l'action qu'il exerce sur son en-dehors, sur ce qui n'est pas lui-mème. I'Influence d'un livre montre l'écriture en tant qu'acte: non pas simplement comme acte d'énonciation ou comme acte propositıonnel mais comme acte illocutionnaire. Le livre, certes, énonce des mots, réfère, prédique ; ce ne sont cependant pas ces actes dont I'Influence d'un livre nous parle. mais d'une capacité d'action qui est de l'ordre de la dimension pragmatique de l'énoncé.

\section{La transformation dé la réalité}

On a souvent noté que la rupture du silence matérialisée par les premiers mots d'un roman concentre au maximum l'intention de dire. ${ }^{3}$ La volonté pragmatique qui caractérise le roman québécois du XIXe siècle pour rait indiquer que cette proposition est valable non seulement pour un roman particulier en tant qu'objet autonome, mais que, lorsque ce roman est le point de départ d'une série romanesque nationale, elle peut s'appliquer également à une partie importante de cette série. En effet, dès que l'on parcourt les textes à travers lesquels les romanciers québécois qui suivent de Gaspé fils présentent et commentent leur propre travail, on s'aperçoit que la référence à une volonté d'influer sur la réalité, d'opérer des transformations concrètes dans l'entourage à travers l'écriture est une constante de ces textes.

Dans sa préface à Jean Rivard le défricheur(1862), Antoine Gérin-Lajoie écrira quiun de ses buts était «d'encourager notre jeunesse canadienne à se porter vers la carrière agricole, au lieu d'encombrer les professions d'avocat, de notaire, de médecin et les comptoirs des marchands, comme elle fait de plus en plus, au grand détriment de lintérêt public et national». "Son personnage deviendra ainsi l'exemplum du Canadien idéal qui préfère a à la vie du lion de ville celle du lion de la forèt». Trois décennies plus tard. Jules-Paul Tardivel donne à son Pour la patrie (1895) un but non moins défini. Il ne s'agit pas pour lu de transformer une tendance malencontreuse du marché du travail mais de s'occuper de la purification des mœurs, raison qui le pousse à défendre un roman q qui fortifie la volonté, qui élève et assainit le ccur, qui fait aimer davantage la vertu et haï le vice, qui inspire de nobles sentiments». C'est à travers ces effets que Tardivel entend contribuer à l'épuration de l'atmosphère qui entoure cette Nouvelle-France dont la raison d'être est l'accomplissement d'une missıon chrétienne en Amérique, et a détruire, si c'est possible, quelquesunes des mauvaises herbes qui menacent de l'étouffer». Non moins évangélisatrices sont les aspirations d'Alphonse Thomas, l'auteur de Albert ou lOrphelin catholique (1885), qui écrit dans la préface de ce roman: * Ce livre traite de questions religieuses, questions de la plus haute importance que tout catholique doit bien comprendre, s'il veut réfuter avec facilité les arguties et les accusations lancées contre notre Église par nos frères séparés. Livre pratique, le roman devra être considéré comme une collection d'arguments dont le lecteur pourra se servir dans le combat contre les protestants. En préfaçant Jeanne la fileuse (1875). Honoré Beaugrand avait donné à son livre un but également pragmatique: "Je n'ai qu'un but en le publiant: celui de rétablir la 
vérité, tout en défendant l'honneur et le bon nom de mes compatriotes émigrés». Cette conception du roman en tant qu instrument du rétablissement de la vérité sera reprise par Edmand Rousseau dans la préface au Château de Beaumanoir (1886): "En face des insinuations malveillantes et des injures qui ont été dites depuis quelques mois contre la population canadiennefrançasse, contre nos milices, il n'est pas de meilleure réponse, croyons-nous. de réfutation plus facile et plus complète, que de rappeler les actions héroiques de nos pères, leur courage dans l'adversité, leur vaillance sur le champ de bataille.

Chez d'autres écrivains, comme Joseph Marmette et Francois-Benjamin Singer, le parti pris pour l'Histoire était déjà venu satisfaire des ambitions beaucoup plus vastes. Ces auteurs se tournaient vers le passé non pas pour y trouver lés éléments servant à donner une réponse immédiate à l'adversaire, mais dans le but de donner aux compatriotes une mémoire collective, de faire revivre des moments épiques qui risquaient de rester ignorés et de se perdre à tout jamais, une façon donc de faire de l'histoire. "Nous devons - écrit Singer dans sà préface aux Souvenirs d'un exilé canadien (1871) - en courageux soldats de l'avenir, lutter contre l'aridité de notre histoire, et nous efforcer de faire jaillir des cendres du cacique. de nos grands lacs et de nos forêts vierges, des récits qui puissent, au moins. aplanir ces difficultés [le manque d'une histoire] à la postérité». Et Marmette, dans l'introduction à François de Bienville (1870): a le but auquel je visais le jour où je traçai les premières lignes de mon livre: rendre plus populaire en la dramatisant la partie héroique de notre histoirem.

Cette volonté didactique aux aspirations plus ambitieuses sera adoptée par Edmond Rousseau dans un autre roman, les Exp/oits d'lberville (1888): "Chaque page de l'Histoire du Canada renferme un drame. Eh bien, dramatisons I'histoire du Canada, faisons mouvoir devant les yeux de notre peuple ces grandes figures de nos annales, avec leurs vertus, leurs passions, et le peuple nous lira». Ce geste "historisant" qui accorde à la littérature une fonction purement didactique trouve un précédent exemplaire dans le prologue à Jacques et Marie. Souvenirs d'un peuple dispersé (1865) de Napoléon Bourassa: Ces pages que j'ai consacrées a [la mémoire de nos pères] et que je vous offre, sant probablement peu de chose: mais si elles peuvent faire verser quelques larmes nouvelles sur les souffrances oubliées de nos parents: si elles servent à retremper nos cœurs dans leur foi et dans leurs vertus de toutes sortes, et nous engagent à imiter leur exemple dans toutes les circonstances difficiles qui sont encore réservées à notre existence nationale, alors je n'aurais pas entrepris une tâche inconsidérée». Le roman se propose ici d'agir non seulement sur la conscience de la collectivité mais, a travers elle, sur le vécu de notre collectivité. II ne s'agit pas de réveiller une mémoire pour y habiter, mais de trouver dans le passé certains modèles propres à être imités dans le présent. Toute idyllique qu'elle soit, même si elle ne fait que favoriser une transformation purement hallucinatoire de la réalité. l'attitude laisse voir derrière le dessein didactique du romancier une volonté de transformation de la circonstance historique contemporaine. 
$\dot{A}$ quelques occasıons. l'intention pragmatique du romancier touche à la littérature elle-mème: on écrira pour encourager d'autres à le faire. Philippe Aubert de Gaspé fils avait fait le geste inaugural en pariant dans la préface à I'Influence d'un livre de sa volonté de faire naître chez ses compatriotes le désir d'enrichir leur pays avec des romans de mœurs. Cette détermination de stimuler la production littéraire acquerra une dimension patriotique chez Joseph Doutre, qui en fait l'objectif majeur aux Fiancés de 1812 (1844): * Notre but principal est de donner quelqu essor a la littérature parmi nous. sı toutefois il est possible de la tirer de son état de léthargie. Nous nous consolerons volontiers des critiques, si l'humilité de notre nom peut faire comprendre à nos jeunes amis qu'ils sont plus capables qu'ils ne le pensent ". Edmond Rousseau. dont la volonté historisante n'est pas moindre que celle de Doutre, partage également avec ce dernier l'aspiration à promouvoir, à travers son travail de romancier. l'essor d'une littérature nationale. Dans la préface déjà citée aux Exploits d'lberville. Rousseau écrit: "Je connais très bien la faiblesse de mes ressources et les proportions très bornées de mon jeune talent. Qui m'empècherait pourtant d'apporter ma pierre, une toute petite pierre, bien humble, à l'édifice? L'idée est généreuse et patriotiqué: qui m'assure qu'elle ne charmera pas les écrivains canadiens, et que nous ne verrons pas avant peu un grand mouvement dans le sens que je viens d'indiquer, mouvement national que j'appelle de tous mes voux».

Modifier les tendances du marché du travail. assainir les mœurs, défendre la religion catholique, rétablir la vérité et défendre l'honneur des compatriotes émigrés, revivre l'héroïque épopée des ancêtres, transtormer les cırconstances historiques immédiates, promouvoir le jaillissement et le développement d'une littérature nationale: pour les romanciers québécois du XIXe siècle, l'activité littéraire a toujours comme but une modification de l'espace extra-littéraire. Le roman est constamment conçu en tant qu'effet sur l'entourage: lorsqu'ils parlent de roman, ces romanciers parlent non d'un livre mais de l'influence d'un livre.

Leur insistance sur l'aspect pragmatique permet d'accorder à tous les énoncés convoqués plus haut le statut d'un lieu commun qu'on pourrait nommer topos de la transformation. La distance qui sépare le jeune de Gaspé du circonspect Thomas, le style boursouflé de Bourassa du ton conversationnel de Rousseau, le libéralisme ardent de Doutre du radical ultramontanisme de Tardivel, $n$ 'empèche les textes préfacièts de ces écrivains d'accueillir tour à tour le mème topos. Par-dessus les hiatus temporels existant entre eux, par-dessus les différences d'âge, de position politique et idéologique, de formation littéraire et d'aspiratıons esthétiques de leurs auteurs, ces textes sont tous d'accord pour affirmer. ne serait-ce que de manière indirecte, que le roman a pour but de modifier la réalité. Ainsi, malgré le poids des divergences, malgré le fait que ces écrits constituent un corpus hétérogène, on peut postuler l'hypothèse que leur argumentation comporte d'autres coincidences du type de celle qu'on vient d'identifier et que, par conséquent, les préfaces, prologues, avant-propos, introductions et postfaces écrites par les romanciers québécois du XIXe siècle se laissent lire, à un certaın niveau, comme des fragments d'un mème discours. 
Retrouver les autres topoi récurrents de ce discours, et leur logique, équivaut à reconstituer une des composantes du discours de l'institution littéraire de la période couverte par ces textes.

\section{La fidélité à la réalité}

Le premier des topoï identifiés a pour objet le rapport du roman avec la réalité. mais il ne touche qu'à l'un des deux sens possibles de ce rapport: la présence du roman au monde. Qu'en est-il du sens inverse? Est-ce que la présence du monde dans le roman est. elle aussi, l'objet d'énoncés ayant un présupposé commun?

"J'ai décrit les événements tels qu'ils sont arrivés, m'en tenant presque toujours à la réalité, persuadé qu'elle doit toujours remporter l'avantage sur la fiction la mieux ourdie». Ainsi s'exprime le jeune de Gaspé fils, formulant une opposition entre "réalité ${ }^{5}$ et fiction que plusieurs de ses successeurs allaient perpétuer, n'accordant toutefois pas toujours le même sort à chacun des termes opposés.

Comme l'auteur de I'Influence d'un livre. Patrice Lacombe situe la réalité au premier plan du roman. Dans sa postface à la Terre paternelle (1846). Lacombe met en relation la vraisemblance de son récit avec la fidélité de l'écriture à l'entourage social tel qu'il le conçoit: "Laissons aux vieux pays, que la civilisation a gâtés, leurs romans ensanglantés, peignons l'enfant du sol tel qu'il est, religieux, honnète, paisible de mceurs et de caractère (...)x. moins rigide que Lacombe, Charles Marcil permettra l'intrusion de la fiction dans l'Heritière d'un millionnaire (1867): "On y trouvera trois espèces de choses: des faits réels, de l'imagination, dans une certaine mesure, et beaucoup de liberté d'alluren. On constate cependant que cette liberté ne touche pas le type d'équilibre proposé par de Gaspé fils: dans la formulation qu'on vient de lire, les faits réels passent d'abord, et sans aucune modalisation, tandis que l'imagination se voit sérieusement admonestée par une locution adverbiale.

Même dans certains cas où elle semble promue au premier rang. l'imagination se voit soumise à une forte dépendance de ce réel privilégié. Bourassa, dans le texte déjà cité, nous en donne un témoignage: "M'étant engagé à faire une ceuvre d'imagination, j'ai cherché au milieu de mes souvenirs, dans les sphères du monde que j'ai le plus connu et le plus aimés.

Quant aux écrivains cultivant la forme du roman historique, ils seront beaucoup plus radicaux face à des éléments purement littéraires dont il est utile de se servir mais qu'il faut absolument tenir en respect. Ainsi, par exemple, Marmette, dans la préface à la première édition de François de Bienville, dira qu'il n'a employé d'intrigue que ce qu'il en faut pour animer le récit. Dans l'introduction à la deuxième édition du mème texte, il dira que dans ses quatre livres, "la fiction n'a que juste assez de place pour qu'on puisse les classer dans la catégorie de romans historiques".

Ces citations, auxquelles on pourrait ajouter plusieurs autres allant dans le mème sens, mettent en évidence le fait que le mode de présence du 
monde réel dans le roman est l'objet, lui aussi, d'un lieu commun récurrent. Ce lieu commun pourrant être baptisé topos de la fidélité à la réalité. Comme on le verra, ses occurrences entraînent souvent, dans l'argumentation des écrivains, l'apparition du topos de la transformation et de références a la dimensıon esthétique ou littéraire du roman.

\section{Transformation du monde, fidélité a la réalité et dimension esthétique}

"Le Canadien n'a pour toute ressource que son imagination. Il faut qu'il soutienne son récit par d'éternelles fictions. Telles sont les causes qui empècheront pour longtemps notre littérature d'avancer aussi rapidement que nous pourrions l'espérer». Elle est bien curieuse cette réflexion de la préface de Souvenirs d'un exilé canadien de François-Benjamin Singer, qui oppose fiction et tittérature. D'après elle, la fiction et l'imagination seraient des catégories incompatibles avec le travail littéraire qui ne devrait s'appuyer que sur les données de la réalité. La réflexion n'est pas seulement curieuse; dans le corpus dont nous nous occupons, elle constitue en fait un hapax: aucun autre des romanciers ne soutient une pareille idée, la plupart d'entre eux tenant plutôt des propos opposés. ${ }^{6}$

Si l'on s'en tient à la dernière citation de Marmette, le recours à la forme romanesque n'obéit pas, pour cet auteur, a un intérêt d'ordre esthétique mais bien plutôt à une nécessité liée aux buts didactiques de son entreprise. On dirait que c'est à contreccur qu'il a recours à cette forme. II semble en fait mettre à profit le goût que pour elle montre une partie de la société, afin de lui faire parvenir un message pédagogique. On imagine volontiers Marmette disant: «puisque le peuple veut du roman moderne, puisqu'il est gâté sous ce rapport, eh bien, donnons-lui du roman; du même coup, nous lui servirons une leçon d'histoire a laquelle il n'aurait pas accès autrement". On pourra peut-être trouver exagérée une telle supposition; ce sont pourtant des paroles d'un autre partisan du roman historique. Edmond Rousseau, qui nous la suggèrent: adramatisons l'Histoire du Canada (...) et le peuple nous lira. Puisqu'il veut du roman moderne. puisqu'il est gâté sous ce rapport, eh bien, donnons-lui du roman, mais vraiment bon, honnête, vertueux, national». Ou ces autres parales d'Honoré Beaugrand dans sa préface à Jeanne la fileuse: "J'ai cru devoir adopter la forme du roman afin d'intéresser la classe ouvrière qui forme aux États-Unis la presque totalité de mes lecteurs $\%$. L'intérèt pour le roman ne vient donc pas de l'écrivain mais du public, d'une commande sociale, et c'est presqu'à son corps défendant que l'écrivain devient romancier. On trouvera alors justifié que Beaugrand continue dans ces termes, qui opposent roman et représentation fidèle de la réalité: «mais je me suis efforcé, en même temps, de faire une peinture fidèle des mceurs et des habitudes de nos compatriotes émigrés. J'ai introduit, en outre, dans man ouvrage, quelques statistiques qui ne sauraient manquer d'intéresser ceux qui s'occupent des questions d'émigration et de rapatriement $*$. On qualifierait difficilement de roman cet ouvrage dont Beaugrand nous parle; il ressemble beaucoup plus à un rapport préparé à l'intention du gouvernement ou à un essai sociologique romancé où les catégories littéraires sont beaucoup moins importantes que ces "renseigne- 
ments authentiques - c'est encore Beaugrand qui écrit - sur la position matérielle, politique, sociale et religieuse qu'occupent les Canadiens de la Nouvelle-Angleterre". Beaugrand lui-mème en était conscient, qui avait commencé la préface à la première édition de l'ouvrage en question avéc cette mise en garde: "Le livre que je présente aujourd'hui au public, sous le titre de Jeanne la.fileuse, est moins un roman qu'un pamphlet: moins un travail littéraire qu' une réponse aux calomnies que l'on s'est plu à lancer dans certains cercles politiques des États-Unis". C'est une mise en garde semblable qu'Antoine Gérin-Lajoie introduit dans l'avant-propos à son Jean Rivard le défricheur: “Ce n'est pas un roman que j'écris, et si quelqu un est à la recherche d'aventures merveilleuses, duels, meurtres, suicides ou d'intrigues d'amour tant soit peu compliquées, je lui conseille de s'adresser ailleurs. On ne trouvera dans ce récit que l'histoire simple et vraie d'un jeune homme sans fortune (...).

Beaugrand et Gérin-Lajoie opèrent, dans les derniers tragments cités, une mise en relation entre le topos de la transformation du monde. le topos de la fidélité à la réalité et la référence à la dimension littéraire du roman. Et leur argumentation, que la plupart des romanciers québécois du XIXe siècle auraient pu endosser sans maugréer, va dans un sens tout à fait inverse à celle de Singer rappelée plus haut. - C'est à cause de mon intention d'intervenir sur la réalité que je ne fais pas un travail littéraire, un roman, mais un pamphlet, dit le premier. - C'est parce que je raconte une histoire vraie que je ne fais pas un roman, dit Gérin-Lajoie. Dans une autre préface au mème ouvrage, cet écrivain sera encore plus transparent: "L'intention de l'auteur (...) n'a jamais été de faire un roman, et il peut assurer que dans les faits et incidents qu'il raconte, il s'est appliqué avec un soin scrupuleux, au risque même d'ennuyer les lecteurs frivoles, à ne rien dire qui ne füt strictement conforme à la réalité *

\section{Le manque de prétention littéraire}

Beaugrand et Gérin-Lajoie pourraient être qualifiés, d'après leurs propres propos, de romanciers contre leur gré. ' Leur aje ne fais pas du roman» ne peut cependant pas être considéré comme un topos propre à l'argumentation de l'ensemble de textes dont nous nous occupons, car bien plus nombreux sont les écrivains qui se présentent aux lecteurs en tant que romanciers par volonté. Il existe toutefois, dans la plupart des textes, un lieu commun qui apparaît comme l'expression euphémique de la déclaration des deux écrivains nommés. Ce lieu commun pourrait être appelé topos du manque de prétention littéraire.

« Je n'ai nullement l'intention de composer un ouvrage scundum artem; encore moins de me poser en auteur classique. - écrit Philippe Aubert de Gaspé dans × La sortie du collègen. chapitre servant de préface aux Anciens Canadiens (1863). pour ajouter plus loin: "J'admettrai franchement qu'il y a mille défauts dans ce livre, et que je les connais. ( ...) J'entends bien avoir, aussi, mes coudées franches et ne $m$ 'assujettir à aucunes règles prescrites. Que les puristes, les littérateurs émérites, choqués de ces défauts. l'appellent roman. mémoire, chronique, salmigondis, pot-pourri: peu m'importe». Le nonchaloir 
un tantinet provocateur de de Gaspé place les Anciens Canadiens dans l'indétermination générıque, geste qui, à travers sa relative équipollence à celui de Beaugrand et de Gérin-Lajoie, confirme par un biais la quasi identité de nature entre la dénégation "je ne fais pas du roman" et l'affichage du manque de prétention littéraire. ${ }^{3}$

« N'ayant aucune prétention littéraire - écrira Alphonse Thomas quelque vingt ans plus tard dans la préface déjà citée -, je sais que ce livre pèche beaucoup par la forme: le lecteur instruit verra qu'il a été écrit par un ouvrier plus habile à manier l'outil que la plume». Napoléon Bourassa avait allégué aussi le manque d'expérience pour justifier les défauts formels de son ouvrage: « N'ayant jamais fait le plus petit volume, ni jamais entretenu l'idée d'en faire un, jai entrepris cet écrit sans forme préméditée, sans modèle adoptén. Le geste sera répété par Tardivel: a Pendant mes vingt ans de journalisme, je n'ai guère fait autre chose que de la polémique. Sur le terrain de combat où je me suis constamment trouvé, j'ai peu cultivé les fleurs, visant bien plus à la clarté et à la concision qu'aux ornements du style. (...) Qu'on ne cherche donc pas dans ces pages le fini exquis des détails qui constituent le charme de beaucoup de romans. Je n'ai pas la prétention d'offrir au public une ceuvre littéraire délicatement ciselée, ni une étude de mceurs patiemment fouillée». Dans d'autres cas, les raisons invoquées seront d'ordre technique, comme il arrive dans la préface à l'Héritière d'un millionnaire de Charles Marcil: «Dans les pages que nous avons écrites, nous ne nous sommes guère soucié des proportions symétriques. (...) N'écrivant pas un poème, nous n'avons pas cru devoir nous soumettre passivement à une discipline littéraire rigoureuse». Quant à Honoré Beaugrand, les précisions dont il fait précéder Jeanne la fileuse mettent encore en évidence la relation intime entre le topos du manque de prétention littéraire et les topoi de la transformation et de la fidélité à la réalité. D'une part, être fidéle à la réalité équivaut à peindre sans artifice les modestes habitudes des personnages; les aspects formels de la narration hériteront de cette nécessité: a L'intrıgue est simple comme les mours des personnages*. D'autre part, l'option en faveur de la mise de l'accent sur la dimension pragmatique suppose la soumission des aspects formels du message aux contraintes de la situation de communication: «j'ai quelques fois sacrifié l'élégance du langage au désir de me faire comprendre des classes ouvrières qui ne lisent encore que bien peu».

\section{La demande d'indulgence}

Ce manque de prétention littéraire, ou plutôt cette manière de reléguer à un deuxième plan la dimensian esthétique est donc conséquence de l'attitude utilitaire, instrumentaliste adoptée par les romanciers face au roman. Devant laalternative de produire des objets utiles ou des objets agréables - la confluence des deux termes étant rarement envisagée - la plupart des écrivains optent pour la première solution. Il est vrai que quelques-uns d'entre eux, à certains moments, déclarent leur intention de procurer au lecteur une certaine jouissance ou quelque chose qui ressemblerait à de la jouissance. Mais le geste, la plupart du temps, est accompli d'une manière qui trahit un vague sentiment de gène, de culpabilité, d'infériorité, ou des trois à la fois. 
Voyons par exemple comment Doutre, tout en la formulant. minimise son intention de satisfaire les attentes de jouissance esthétıque du lecteur: "Car nous avons la petite présomption d'avouer que si nous n'eussions pas eu l'espoir de plaire quelque peu, nous ne publierions pas notre essai». C'est là une des rares fois où un romancier québécois du XIXe siècle ose faire référence a de pareilles velléités. Chez les autres, en général - rappelons par exemple l'ennui que Gérin-Lajoie se déclare prêt à infliger au lecteur --. l'option pour le prodesse éclipse complètement la possibilité d'existence du delectare. La fin ne jugeant pas mais justifiant les moyens, on peut se permettre de ne pas être agréable - d'être même désagréable - lorsqu'il s'agit, d'abord et avant tout, d'être utile.

Mais une fois l'option faite et exposée, parfois mème avec vantardise, un brin de commisération pour le lecteur semble jaillir de manière soudaine. Tout arrive comme si les romanciers se sentaient subitement coupables d'offrir au lecteur un livre dont l'impraticabilité semble, dans leur conscience, tenir de l'ordalie. C'est ce qui explique, du moins partiellement, que la formulation du manque de prétention littéraire soit suivie, dans la majorité des cas, d'un autre lieu commun qu'on peut nommer topos de la demande d"indulgence et dont un premier exemple nous est ici donné par la lettre servant de préface à Une apparition (1860) d'Eraste d'Orsonnens. Après avoir qualifié son ouvrage d'essai d'écolier, après avoir signalé qu'il y voit de grands défauts, que xles matériaux $n$ y sont pas disposés dans le meilleur ordre et que les personnages y sont trop nombreux, quoique tous nécessairesn, l'auteur se permet de noter qu'il espère qu'Une apparition "sera accueillie avec indulgencen. Charles Marcil, dans le texte déjà cité. mettra en relation la demande d'indulgence avec son geste d'affranchissement par rapport aux règles formelles: “Nous avons laissé courir notre plume librement, plus anxieux de plaire par les détails que par les combinaisons d'ensemble. On sera assez bienveillant pour nous tolérer cet acte d'émancipation». Philippe Aubert de Gaspé fils avait déjà allégué la valeur inaugurale de son roman pour justifier la demande au lecteur d'une pensée consolante pour l'auteur: a J'offre à mon pays le premier roman de mceurs canadien, et en le présentant a mes compatriotes je réclame leur indulgence à ce titre ». Pour sa part, Patrice Lacombe invoquera solennellement les motifs élevés quil'ont amené a écrire afin de solliciter le pardon pour les défauts de son ouvrage. Quant à Singer, lorsqu'il s'agira de formuler l'inévitable pétition, suivant encore une fois son élan lyrique, il fera confiance aux charmes indiscutables de l'octosyllabe:

O cher lecteur, si je t'ennuie

Pardonne-moi, je t'en supplie...

Pense au héros, non a l'auteur,

Accorde une larme au malheur!!!

Moins vagues et insaisissables seront les raisons données par Honoré Beaugrand qui renvoie le lecteur aux conditions de production de son livre: aÉcrit au jour le jour, publié en feuilleton et mis en pages immédiatement, sans être révisé, cet ouvrage a droit à l'indulgence que l'on accorde généralement aux articles de journaux. Ce que je demande de la bienveillance du lecteurn. 


\section{Dit et non-dit de l'argumentation}

On pourrait mettre fin à cette enquète, considérant qu'elle nous a permis de reconstruire, à travers l'étude du discours tenu par les romanciers sur leurs propres romans, l'une des composantes du discours de l'institution littéraire québécoise au XIXe siècle. D'après ce discours, la constitution, à la suite de l'échec de la Rébellion de 1837-38, de ce que René Dionne a très heureusement nommé "la patrie littérairen demande un roman dont la fonction est d'opérer des transformations concrètes dans la réalité. Cet engagement a une contrepartie dans le parti-pris en faveur d'une représentation fidèle de la "realité, Le double choix a des conséquences sur le plan esthétique: tout ce qui est de l'ordre de l'artifice, de la fiction, du «littéraire» se trouve en contradiction avec la représentation et l'effet recherchés. Ainsi, l'écrivain devra parfois se défendre d'être romancier et, plus généralement, de faire de la littérature. Finalement, dans la mesure où il a conscience du fait que les lecteurs recevront malgré tout le produit de son travail comme un objet littéraire, il leur demandera d'être miséricordieux et de pardonner les défauts dont cet objet souffre sur le plan esthétique.

La reconstruction que nous venons de faire est pourtant incomplète. En fait, elle ne s'appuie, pour l'essentiel, que sur le dit du discours des romanciers du XIXe siècle, et laisse très sauvent de côté tout ce qu'il peut y avoir dans ce discours de déplacements argumentatifs. Revenons donc, pour que nous restions bien conscients du caractère inachevé de ce travail mais également pour faire peut-être un autre pas en avant, sur les réflexions faites plus haut autour du dernier des topoï que nous avons identifiés, celui de la demande d'indulgence, qu'elles ne paraissent pas expliquer complètement. Le caractère systématique de cette demande et, surtout, le rapport contradictoire qu'elle maintient avec l'ostentation d'un manque de prétention littéraire incitent à chercher d'autres motifs que ceux qu'on a établis en suivant le raisonnement des énonçants, des motifs plus subreptices qui, malgré cela - ou peut-être à cause de cela - pourraient être considérés comme déterminants.

La recherche de ces motifs s'inspire ici de l'hypothèse selon laquelle la littérature québécoise arrive à dire à travers et malgré les normes régulatrices de l'institution littéraire française ${ }^{9}$. II est évident que cette hypothèse ne peut être mise à l'épreuve qu'à travers un travail d'analyse des textes romanesques eux-mèmes. Toutefois, une lecture du discours tenu par les romanciers québécoıs du XIXe siécle sur leurs propres romans - une lecture qui s'attarderait beaucoup plus à ce que ce discours ne dit pas qu'à ce qu'il dit - paraît être appelé à en renforcer la pertinence.

Pour le romancier québécois du XIXe siècle, le roman, en tant que forme littéraire, est essentiellement français. Souvent réfractaire à employer le substantif pour désigner le produit de son propre travail et celui de ses compatriotes, il lui préfère les mots essai, ouvrage, histoire, récit, narration, etc. De la mème manière, les règles d'écriture du roman sont pour lui les règles régissant la production romanesque française. On a vu, également, avec quelle 
force il rejette ces règles. Or ce romancier parle souvent de roman et de règles d'écrıture. ce qui est en quelque sorte paradoxal. Concentrons-nous un moment sur les références au roman. La tradition est inaugurée par Philippe Aubert de Gaspé fils qui se plaint du fait que le Canada, "pays vierge, encore dans son enfance, $n$ 'offre aucun de ces grands caractères marqués, qui ont fourni un champ si vaste au génie du romancier de la vieille Europe". On sait que cette perspective méliorative du romancier européen - lire : français - sera vite abandonnée et corrigée avec application gràce à l'exercice d'une violence verbale se voulant parfois fulminante. "Laissons aux vieux pays, que la civilisation a gâtés, leurs romans ensanglantés " dira Lacombe avec un dédain qui cache mal un germe d'animosité dont la manifestation sans ambages aura lieu chez des auteurs comme Rousseau et Tardivel. Bien que les raisons morales soient toujours à la base de ces condamnations sans appel, le dénigrement rẻitéré du roman français, de Lacombe à Tardivel, pourrait ne pas être entièrement motivé par ce genre de raisons. Le très libéral Joseph Doutre. dans sa préface aux Fiancés de 1812 - préface qui reprend par ailleurs, dans une perspective originale, toute une discussion, en vogue à l'époque, sur les aspects éthiques de la production romanesque - n'occulte pas son malaise face à un apréjugé en faveur de l'étranger» qu'il présente, dans le domaine littéraire, comme exagérément généralisé au moment où il écrit. Doutre arrivera même, faisant référence aux "francisants", à lancer cette mise en garde: "Les fiancés ne sont pas écrits pour ces messieurs. Le cœur leur en soulèverait de dégoût. Ainsi se garderont-ils d'y toucher ».

Les attaques contre la France, et plus particulièrement contre sa littérature, peuvent ainsi, dans une certaine mesure, se voir justifiées par l'attachement qu'à l'endroit de cette littérature exhibe un groupe considérable de lecteurs canadiens. Pourquoi exclure de ce groupe quelques écrivains dont les critiques acerbes paraissent fréquemment venir cacher un sentiment d'admiration de l'autre et de mépris de soi-même? Singer nous donne un exemple de ce double sentiment sous la forme d'un éloge a Rousseau : « JeanJacques Rousseau a fait des confessions, mais bien différent du philosophe genevois qui écrivit sa vie après avoir rempli l'univers de ses chefs-d'ceuvre. je viens, moi. le plus déshérité de la création et inconnu au monde littéraire (...). . Et dans le paragraphe suivant: "Qui suis-je relativement aux grands génies dont je veux suivre la trace? Un atome imperceptible perdu au milieu de la créations.

II ne semble pas trop aventureux de dire que, malgré la profusion de condamnations dont elle est l'objet, condamnations qui fonctionnent en vérité très souvent comme dénégations - au sens psychanalytique de ce terme -. la littérature française hante la conscience du romancier québécois du XIXe siècle, et que cette littérature est constamment en train de faire pression sur son activité scripturale. Le roman et les codes littéraires français constitueraient ainsi une sorte de modèle auquel le romancier voudrait secrètement çonformer le produit de son travail.

La puissance de ce modèle a des raisons historiques. Pour cet écrivain, issu d'une petite bourgeoisie professionnelle qui, après 1837-38, ne trouve 
d'autre moyen, pour s'opposer au vainqueur, que la résistance défensive. l'activité littéraire est devenue une pratıque vouée à l'aider à définir son identité individuelle. sociale et nationale. Cette définition de son identité, il essaıera de l'atteindre à travers deux mouvements complémentaires. D'une part, l'opposition a l'adversaıre qui se manifeste sur le plan thématique du roman à travers des catégories comme l'Anglais, le marchand, le protestant, l'industrie, la ville, etc. D'autre part, l'identification à la francité qui se manifeste sur le plan thématique également, à travers des catégories comme le passé épique, la mémoire des ancêtres. la langue, la campagne, la religion, etc., mais, surtout. sur le plan formel à travers les codes littéraires français.

II serait intéressant de suivre la trace des modalités prises par ce jeu d'oppositions et d'identıfications depuis les débuts du roman québécois jusqu'a nos jours. Une telle enquète nous éclairerait peut-être sur la façon dont une littérature matérialise et manifeste, à travers son histoire, les avatars du mouvement d'une collectivité vers son émancipation. Nous appuyant sur une réflexion de Gramsci à ètre repensée par rapport à un contexte socio-historique plus restreint que celui auquel elle fait allusion - u toute nouvelle civilisation, dans la mesure où elle en était une, mème comprimée, combattue et verrouillée de toutes les manières, s'est exprimée littérairement avant que dans la vie étatique, de sorte que son expression littéraire a été le moyen de créer les conditions intellectuelles et morales pour l'expression législative et étatique „10 - nous pourrions avancer qu'une telle enquète nous éclairerait également sur la manière dont la série romanesque québécoise annonce, prépare et participe à la transformation de l'ordre social.

1. Les propositions faites dans cet article ont été développées dans le cadre d'un sémınaire sur le discours de l'instıtution littéraire québécoise tenu à l'UOAM à l'automne 1981, sous la direction de Jacques Allard.

2. Le mouvement littéraire au Canada*, in le foyer canadien. IV, 1866. Cité par Denis Monière, le Développement des idéologies au Québec des origines à nos jours. Montréal, Éditions Québec/Amérique, 1977. p. 182.

3. Voir par exemple: Jacques Leenhardt, Lecture politique du roman. La jalousie d'Alain Robbe-Grillet, Paris, Minuit, 1973, p. 47, d'où nous tirons la formulation.

4. Toutes les citations sont tirées du recueil de Guldo Rousseau : Préfaces des romans québécois du XIXe siec/e. Sherbrooke. Cosmos, 1971. Compte tenu de la brièveté des textes constituant ce recueil, nous n'avons pas cru nécessaire d'indiquer la pagination des fragments cités.

5. Afin de ne pas surcharger notre texte de guillemets, nous n'entourerons pas toujours de ce signe typographique le mot aréalité " qui en aurait pourtant grand besoın. On sait jusquà quel point la représentatıon de la réalité, entendue de manière classique. est absente des romans dont Il est ici question. Au demeurant. dans le présent travail, nous ne nous occupons pas de ces romans, mais d'un discours tenu sur eux.

6. Il faut néanmoins signaler qu'il semble que l'idée formulée par Singer jouissait a l'époque d'une certaine popularité et avait bonne presse. Dans une note publiée dix-neuf ans après la parution de Souvenirs d'un exile canadien on peut lire: - Nous avons beau dire, cest la fiction qui a détérioré notre tempérament intellectuel, c'est elle qui a retardé notre croissance littéraire. A force de nous assimiler les contes et nouvelles que nous servent quelques désceuvrés de Paris, nous avons perdu le goüt et l'appétit des études sérieuses". La Revue canadienne, troısième série. III (1890); cité par Yves Dostaler. les infortunes du roman dans le Québec du XIXe siècle, Montréal, Hurtubise, HMH, 1977. p. 117. 
7. On hésite à classer Tardivel dans cette catégorie. même si quelques-unes de ses réflexions paraissent le justifier. On se rappellera ses invectives contre le roman. * cette terrible invention* qu"il faudrait détruire "pour le bien de lhumanité; car. les suppôts de Satan le feront toujours servir beaucoup plus à la cause du mal que les amis de Dieu n'en pourront tirer d'avantages pour le bien*. Mais tout cela n'empèche pas Tardivel de devenir lui-même romancier: "pour fivrer le bon combat - dira-t-il _. il faut prendre toutes les armes, mème celles qu'on arrache à l'ennemix. Et plus loin: *Toute satanique que peut être son origine lon peut se servir du roman ] ad majorem Dei gloriam - Ce que l'auteur prétend faire en écrivant Pour la patrie. On trouve des diatribes semblables contre le roman chez Rousseau.

8. On se rappellera que Philippe Aubert de Gaspé fils ouvre la préface de I'Influence d'un livre par un rejet du Cours de litterature de Laharpe et des règles d'unité chères à la critique du XVIlle siècle. On ne peut pourtant pas parler dans ce cas d'ostentation d'un manque de prétention littéraire, car le rejet est accompli au nom de la *modernitém. Nous laissons volontairement de côté ici le problème posé par les multiples contradictions logiques et erreurs conceptuelles de cette préface, dont la considération pourrait éventuellement conduire à voir, dans le dit rejet, un geste comparable à celui d'autres romanciers québécois du XIXe siècle.

9. Elle a été formulée par André Belleau: «e conflit des codes dans l'institution littéraire québécoisen, in Liberté n 134, mars-avril, pp. 15-20.

10. Antonio Gramsci, « Contenutisti * e *alligrafi *x, in Marxismo e letteratura la cura di Giuliano Manacorda). Roma, Editori Riuniti, 1975, p. 110. 